\title{
USING DJI PHANTOM 4 RTK DRONE FOR TOPOGRAPHIC MAPPING OF COASTAL AREAS
}

\author{
Y. Taddia ${ }^{1, *}$, F. Stecchi ${ }^{2}$, A. Pellegrinelli ${ }^{1}$ \\ ${ }^{1}$ Engineering Department, University of Ferrara, via Saragat 1, 44122 Ferrara, Italy - (yuri.taddia, alberto.pellegrinelli)@ unife.it \\ ${ }^{2}$ AdriaRilievi, via Castel San Pietro 54, 48121 Ravenna, Italy - fstecchi@adriarilievi.com
}

KEY WORDS: UAV, direct georeferencing, on-board RTK, coastal mapping, Structure-from-Motion.

\begin{abstract}
:
Imagery acquisition systems by Unmanned Aerial Vehicles (UAVs) have been rapidly evolving within the last few years. In mapping applications, it is the introduction of a considerable amount of Ground Control Points (GCPs) that enables the final reconstruction of a real-scale framed model. Since the survey of GCPs generally requires the use of total stations or GNSS receivers in Real Time Kinematic (RTK), either with or without a Network approach (NRTK), this on-site operation is particularly time consuming. In addition, the lack of clearly image-recognizable points may force the use of artificial markers (signalised GCPs) whenever no features are naturally available in the field. This implies a real waste of time for the deployment of the targets, as well as for their recovery.

Recently, aircrafts' manufacturers have integrated the on-board RTK capability on their UAVs. In such a way, the high precision GNSS system allows the 3D position detection of the camera at the time of each capture within few centimetres. In this work, we tested the DJI Phantom 4 RTK for the topographic survey of a coastal section in the Northern Adriatic Sea (Italy). The flights were performed flying at an $80 \mathrm{~m}$ altitude to ensure a Ground Sample Distance (GSD) of about 2 centimetres. The site extended up to 2 kilometres longitudinally. The results confirm that the on-board RTK approach really speeds up the precise mapping of coastal regions and that a single GCP may be needed to make a reliable estimation of the focal length.
\end{abstract}

\section{INTRODUCTION}

In the last years, the acquisition of aerial imageries by Unmanned Aerial Vehicles (UAVs) has experienced a rapid increase. Very high resolution imageries acquired up to one hundred meters distance from the ground ensure the reconstruction of detailed models. Even though the absolute scale of such models may be determined using known distances, for many land mapping applications the reference system is generally as important as the scale.

Whenever a precise georeferencing is required, the use of Ground Control Points (GCPs) enables to perform an Helmert transformation by both considering a roto-translation and a scale factor. Modern computer vision algorithms perform a Bundle Block Adjustment (BBA) estimating both the interior and exterior camera orientation parameters using the tie points matched against two or more images and the GCPs. Although tie points give additional constraints for the BBA, the use of GCPs is generally a more robust way to ensure the production of a reliable model. In order to achieve the best accuracies, some authors (Martínez-Carricondo et al., 2018) point out that GCPs should be located around the edges of the surveyed area for best horizontal accuracies, while a stratified distribution is needed for a vertical control.

Direct georeferencing (Gabrlik, 2015, Gabrlik et al., 2016) is an alternative way for the precise reconstruction of models framed within a given reference system. This technique makes unnecessary (Rabah et al., 2018) the use of any GCP. The use of on-board GNSS receivers either in RTK or NRTK mode enables the precise collection of the drone's position at the time of each capture. It is therefore crucial for such systems to

\footnotetext{
${ }^{*}$ Corresponding author
}

promptly and accurately record the instant in which the image was captured, possibly in the same time reference of the GNSS data (typically GPS time). Thanks to a calibration (Heipke, 2000, Cramer, Stallmann, 2001) is then possible to compute the camera location applying corrections for the offset between the Antenna Phase Centre (APC) and the camera's perspective centre. Tilt of the drone is also taken into account thanks to an on-board Inertial Measurement Unit (IMU).

The recent availability of commercial drones with an integrated dual-frequency multi-constellation (GNSS) receiver with decreasing costs makes the direct georeferencing approach a more affordable way to conduct aerial surveys by UAVs. Indeed, the higher cost of the overall system in comparison to standard drones is counterbalanced by the time saved for the GCPs surveying. In addition, the speed-up of data acquisition in the field makes this approach more productive. This latter aspect is crucial whenever large extents have to be mapped by means of UAVs with the highest accuracy and as fast as possible. In addition, the direct georeferencing of the images with high accuracy is essential whenever inaccessible, safeguarded or even hazardous areas have to be mapped.

For all the above reasons, in this work we decided to test the novel DJI Phantom 4 RTK for the topographic mapping of a coastal stretch. The recognition of coastal areas of the Emilia-Romagna region (Italy) covers about $120 \mathrm{~km}$ of shoreline and hence it is crucial to use a mapping technique that combines a quick data acquisition and reliable final products.

In particular, this article highlights the accuracies achievable with surveying and processing strategies that differ each other (Padró et al., 2019) for the use or not of some GCPs, for the camera calibration assumptions, for the use of nadiral or oblique images and for the accuracy specified for the RTK 
camera locations and it seeks to identify the best compromise in term of time spent in the field and final accuracy of the model.

\section{MATERIALS AND METHODS}

In order to investigate the accuracies achievable with an on-board RTK approach for the topographic mapping of a coastal section, a DJI Phantom 4 RTK aircraft (Fig. 1a) has been used to perform all the aerial imagery acquisitions. Thanks to its own base receiver, the D-RTK 2 Mobile Station (Fig. 1b), it was therefore possible to precisely georeference the position of the aircraft at the time of each photograph's capture. Consequently, the camera location may be estimated applying corrections for both the offset between the APC and camera's perspective centre and the tilt of the drone: this task is autonomously performed by the firmware and the coordinates stored within the Exif metadata are already referred to the camera.
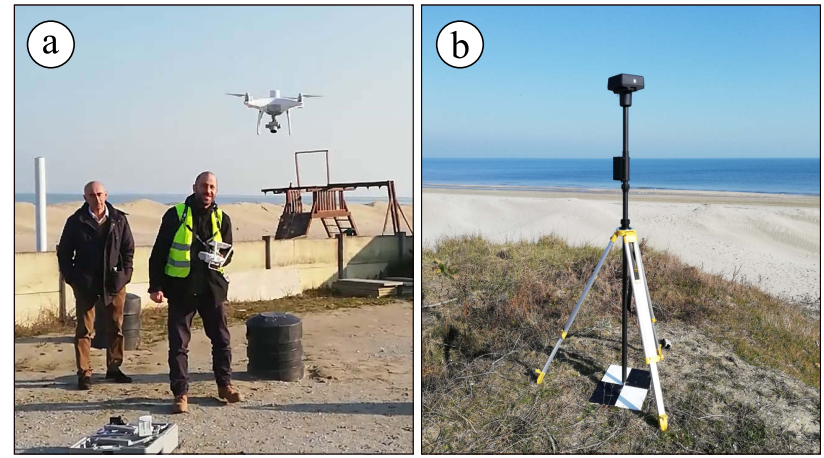

Figure 1. (a) DJI Phantom 4 RTK during landing operations; (b) The D-RTK 2 Mobile Station.

The coordinates set up for the base station ensure that the entire model will be framed in the same reference system of those coordinates. Thus, in all the following tests, the D-RTK 2 Mobile Station was set up on a target whose position was previously defined by a 30 -seconds NRTK surveying. The NetGEO service (TopCon Positioning Italy) was used for the application of differential corrections transmitted in real time from a network of continuously operating reference stations framed in a national reference system (Shen et al., 2015). The coordinates were thus imposed to the D-RTK 2 Mobile Station by setting the latitude, longitude and height. This latter was already accounted for the instrument height.

The site selected for the tests lies along the coast of the Emilia-Romagna region (Italy). It extends up to $2 \mathrm{~km}$ in the North-South direction between the towns of Marina Romea and Porto Corsini, in the Province of Ravenna (Fig. 2). This coastal stretch is particularly suitable for the tests conducted by using a RTK-capable drone because of the wide extent in length and the relatively limited width and it is well representative of the overall coastal morphology that can be found along the Emilia-Romagna shoreline. Moreover, the base-rover distance up to $1.2 \mathrm{~km}$ allowed us to assess the maintaining of fixed RTK solutions in coastal mapping operating conditions. Table 1 reports the range and the accuracy of the system composed by the DJI D-RTK Mobile station and DJI Phantom 4 RTK.

Before proceeding with the imagery acquisition by flight missions, a set of control points was deployed on the beach by the signalization of crosses (Fig. 3), the centre of each
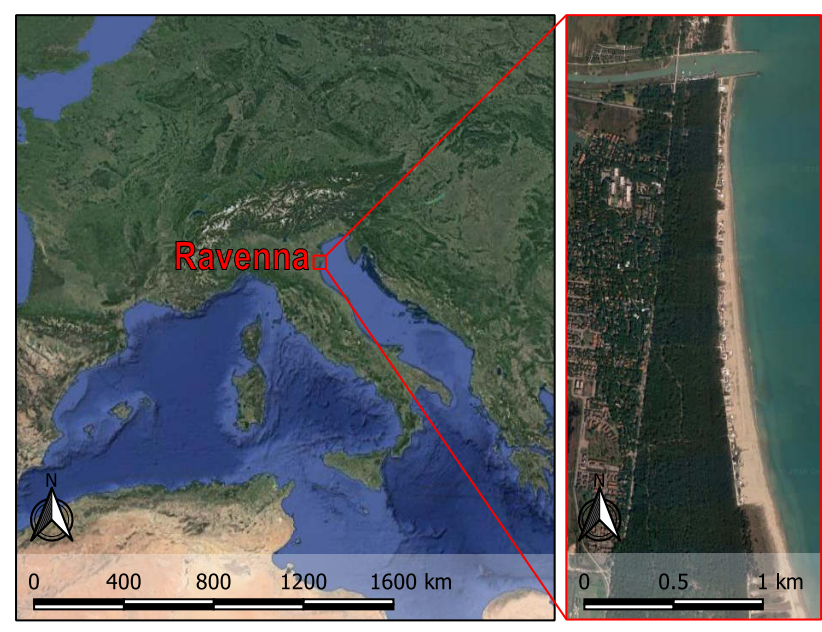

Figure 2. Case study location.

Table 1. DJI system specifications.

\begin{tabular}{cc}
\hline \multicolumn{2}{c}{ DJI D-RTK Mobile station + DJI Phantom 4 RTK } \\
\hline Communication Distance & up to $2 \mathrm{~km}$ \\
RTK horizontal position accuracy (RMS) & $1 \mathrm{~cm}+1 \mathrm{ppm}$ \\
RTK vertical position accuracy (RMS) & $2 \mathrm{~cm}+1 \mathrm{ppm}$ \\
\hline
\end{tabular}

one was hence surveyed through a GNSS geodetic receiver in NRTK mode (the same used to determine the position of the target on which the base station was established). About 40 control points (Fig. 6) were surveyed along the entire coastal section, approximately spaced each other by $100 \div 150 \mathrm{~m}$. In critical areas, such as along the shoreline, control points were signalized as a couple of independent crosses, in order to ensure the preservation of a single one at least. The previously mentioned value of 40 control points thus actually refers to all of the signalised cross, including double ones. The control points were further considered as actual GCPs or, in some cases, as check points (CPs) to validate the accuracy of the model reconstruction. Coordinates of the control points were collected within the official Italian reference system, corresponding to the European Terrestrial Reference System ETRS89 in its ETRF2000 (2008.0) realization. The ITALGEO 05 geoidal separation model was finally used to convert ellipsoidal heights of the GCPs/CPs to orthometric elevations, while geographical coordinates where projected in cartographic UTM-zone 32 Eastings and Northings.

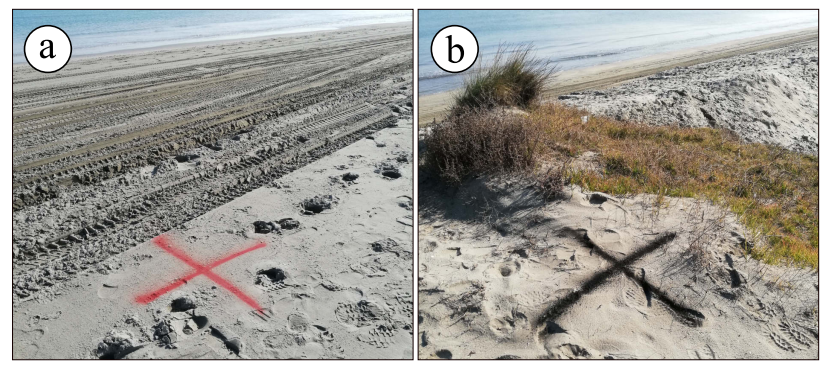

Figure 3. Ground Control Points signalisation:

(a) on the shoreline, (b) on the back-dunes.

Once that GCPs and CPs were signalised and surveyed, different flight missions were thus planned and executed. In particular, the first one consisted in the acquisition of images following a single-grid plan, thus collecting pictures along one 
direction only. In this case the dataset was made up of nadiral imageries and the entire coastal section was mapped, in its $2 \mathrm{~km}$ extents. Due to the limited autonomy of the DJI Phantom 4 RTK, the mission was interrupted and thus resumed with a second battery. This aspect is automatically accounted by the flight planner and does not represent an issue for the mapping of large extents in coastal applications. A second flight mission was performed using a double-grid plan on a smaller region of the previous area. In this case the images were acquired along two orthogonal directions and the camera was set up with a pitch angle of $30^{\circ}$. The number of acquired images, covered extents and other flight plans parameters are summarised in Table 2.

During the in-field operations, an independent set of points was also surveyed with the same GNSS receiver in NRTK mode. These validation points (VPs), not signalised on the beach, were collected directly on the sand (Fig. 4) by using a plate at the lower pole's end. Thanks to this foresight, it was possible to prevent vertical biases due to the sinking of the pole during survey operations. The amount of these point was 119 in total and 98 of which were in common between the nadiral and oblique imageries flight plans.
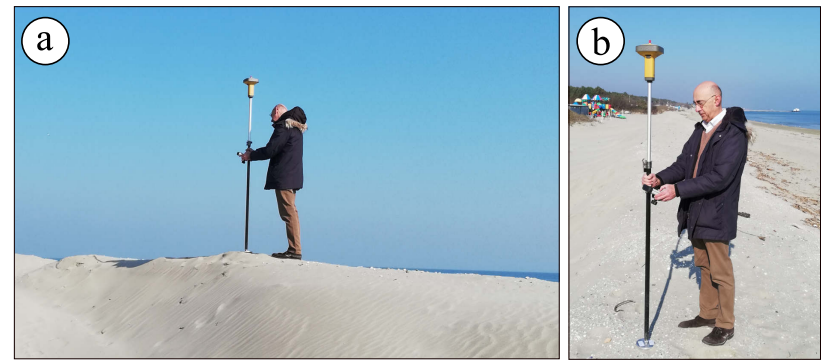

Figure 4. GNSS survey of validations points:

(a) on the top of the dunes, (b) on the beach.

After their acquisition, all the data were processed through the Agisoft Metashape Professional software to align the imageries. This software represents the newest version of the popular Agisoft Photoscan Professional.

In order to avoid differences in the data processing due to the manual recognition of the targets on each image in which they appear, this task was performed beforehand, thus prior to proceeding with the final estimation of the exterior and interior orientations. A template project was saved and modified afterwards. The "reset alignment" option was used to re-initialise all the orientation estimations. In this way the targets' specification on the images became an invariant of the subsequent data processing performed and did not affect the results. Figure 5 shows one of sparse clouds (tie points) generated for the oblique imageries dataset.

Table 2. Flight plan specifications.

\begin{tabular}{ccccc}
\hline Type & Alt. & Img. & Surface ext. & Shoreline ext. \\
\hline Nadiral & $80 \mathrm{~m}$ & 723 & $0.367 \mathrm{~km}^{2}$ & $2.2 \mathrm{~km}$ \\
Oblique & $80 \mathrm{~m}$ & 612 & $0.129 \mathrm{~km}^{2}$ & $1.1 \mathrm{~km}$ \\
\hline
\end{tabular}

Using the nadiral imageries dataset, a total of 12 projects was made. Parameters that were varied consisted in:

- setting a global accuracy for the RTK coordinates equal to $5 \mathrm{~cm}$ or using the Exif recorded value;
- using no GCP with either a known Exif-provided calibration or performing a self-calibration;

- using a set of GCPs with either a known Exif-provided calibration or performing a self-calibration;

- using only a single GCP either barycentric or perimetric and performing a self-calibration.

Similarly, the same 12 projects were made with the oblique imageries dataset. In order to have a compact notation for the further presentation of the results, the following abbreviations will be used in Table 3 and Table 4:

$$
\begin{gathered}
\begin{array}{c}
\text { Ground } \\
\text { Control } \\
\text { Points }
\end{array} \begin{cases}\mathrm{N} & \text { not used } \\
\text { 1B } & \text { one, barycentric } \\
1 \mathrm{P} & \text { one, perimetric } \\
\mathrm{Y} & \text { well }- \text { distributed set }\end{cases} \\
\begin{array}{c}
\text { Camera } \\
\text { Calibration } \\
\text { Method }
\end{array}
\end{gathered} \begin{cases}\mathrm{S} & \text { self }- \text { calibration } \\
\mathrm{E} & \text { Exif provided parameters }\end{cases}
$$

For both nadiral and oblique imageries and for both global and Exif recorded camera accuracies, the considered cases were respectively: N-S, N-E, 1B-S, 1P-S, Y-S, Y-E.

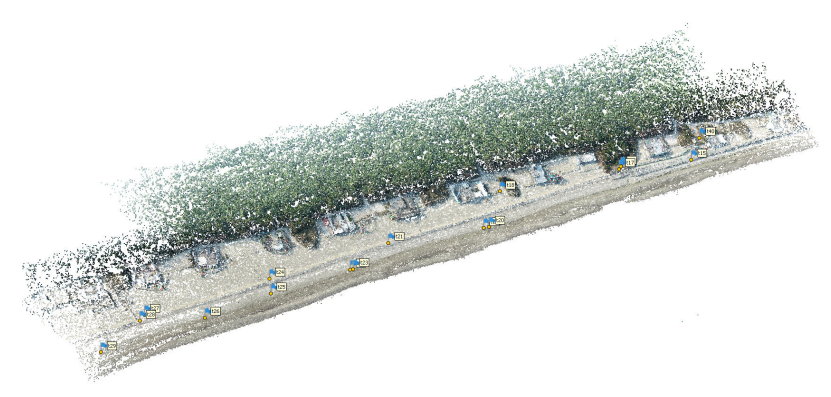

Figure 5. Oblique imageries tie points

For those projects that provided an advantage in terms of simplification of the work (e.g. the use of a single GCP instead of set of GCPs), still ensuring a good accuracy of the final results, a Digital Elevation Model (DEM) was also created to additionally assess the actual vertical precision of the model. VPs were thus finally used for the final computation of elevation differences with the expression:

$$
\Delta H=H_{G N S S}-H_{D E M}
$$

\section{RESULTS}

The data processing conducted by Agisoft Metashape Professional allowed the accurate estimation of the camera locations (exterior orientation) and the camera model's parameters (interior orientation) by performing a BBA and a self-calibration procedure. Variations within this workflow were represented by setting different camera position accuracies ( $5 \mathrm{~cm}$ global or the Exif recorded value) and using a known camera calibration stored in the Exif metadata of the images. Also the number of GCPs to be considered for the alignment of the images was varied. 
The assessment of the overall alignment accuracy was conducted first on the basis of the residuals computed on both the GCPs (if used) and the CPs. All the surveyed targets that were not considered as GCPs in a project, were consequently assumed as CPs within the same project. The residuals computed on both the GCPs and the CPs clearly showed significant differences in the accuracy of the models obtained by varying georeferencing strategies.

The results of all the different processing methods undertaken for the nadiral imagery dataset, assuming a global accuracy of $5 \mathrm{~cm}$ for the camera accuracy, are reported in Table 3. The table shows the residuals computed on the CPs and, if any, the residuals computed for the GCPs after the estimation of the exterior orientations. This latter are reported in italics within brackets.

The lack of GCPs with a dataset of nadiral imageries implied a vertical offset of about $2 \mathrm{~m}$ for the entire model. This issue is well-known in the literature, as also recently investigated by some authors (Forlani et al., 2018) and it is caused by a wrong estimation of the focal length within the self-calibration procedure. Indeed, this latter method is able to model the lens distortion, finally providing good horizontal residuals on all the CPs even with no GCPs, but it fails in calculating the principal distance and thus the model's points are positioned lower or higher with respect to their actual location whenever the focal length is estimated longer or shorter. This fact is due to the practically fixed cameras' altitudes in an RTK approach with high camera accuracies set.

Since a camera calibration is included within the Exif metadata of every DJI Phantom 4 RTK image, it would be therefore reasonable and convenient to fix all those parameters (the so-called "DewarpData" field contains them). In particular, also the focal length becomes known and unbiased in this way. In spite of the assumption made, this approach did not provide a significant advantage for the datasets used in this work. The interior orientation parameters estimated by the self-calibration using a well-distributed set of GCPs significantly differed from the ones of the Exif metadata and hence the model was affected by high errors in all the $x, y, z$ components and not only a bias in the $z$ direction, even though vertical residuals were found to be smaller thanks to a more reliable focal length (imposed) value.

The introduction of a single GCP with nadiral imageries represented an additional constraint that proved to be sufficient (Forlani et al., 2018) in order to make a consistent and reliable estimation of the focal length. Indeed, whenever a GCP is considered, the root mean square error (RMSE) of the residuals computed on the check points with the self-calibration procedure decreased to few centimetres. The expression of RMSE is:

$$
R M S E=\sqrt{\frac{\mathbf{v}^{\mathrm{t}} \mathbf{v}}{n}}
$$

where $\mathbf{v}$ represents the vector of the residuals and $n$ it is the number of its elements.

It is interesting noticing that no significant differences are recognizable on both the horizontal and vertical controls when a barycentric GCP or a perimetric one were used. However, the assessment of the actual vertical accuracy of the DEM, conducted by the computation of frequency histograms with $2 \mathrm{~cm}$ wide classes, shows that a bias of $4 \mathrm{~cm}$ exists between the two strategies (Fig. 7).
It is worth noting that the standard deviations in both cases have the same magnitude, again with a $4 \mathrm{~cm}$ value, while considering a well-distributed set of GCPs leads to a $2 \mathrm{~cm}$ mean value of the discrepancies. The same GNSS receiver in NRTK mode was used for the survey of both the GCPs and the VPs. In addition, the set of VPs was acquired for the entire duration of the in-situ operations, while the signalised GCPs and CPs were surveyed only prior to the flight missions execution. This fact is important because a network service was used for the application of differential corrections to the GNSS-observables: biases or drifts may thus have affected the actual accuracy and precision of the overall set of VPs. However, this did not not represent a serious issue, even though has to be considered whenever analysing and commenting the results obtained by the comparison on VPs.

Similarly to what presented above, the results of all the different processing methods undertaken for the oblique imagery dataset, assuming the Exif recorded data for the camera accuracy, are reported in Table 4.

It is worth noting that using oblique imageries the lack of GCPs did not affect the accuracy of the alignment. Indeed, a reliable estimation of all the internal orientation parameters, thus including the focal length, is already provided through this strategy. The residuals computed on the CPs are completely comparable with the Structure-from-Motion approach with a $2 \mathrm{~cm}$ Ground Sample Distance (GSD) and the NRTK technique used for the survey. In order to analyse all the possible strategies, also the results obtained by use of a single (barycentric or perimetric) GCP were investigated, as well as the use of a comprehensive set of well-distributed GCPs. However, no significant advantages were found. Moreover, the imposition of interior orientation's parameters with the oblique imagery dataset led to a remarkable decreasing in term of precision of the entire model with respect to the case with a self-calibration procedure. This was caused by the same reasons mentioned for the dataset of nadiral imageries: a focal length significantly different from the one estimated through the project with both a set of GCPs and the self-calibration. The DEM was thus computed for those cases that proved to be efficient for the time needed in the field (no GCP used) and the ones representing the best commonly used approach (use of a set of GCPs).

The results of the final assessment of the models' accuracy, conducted on the basis of the VPs, is reported in Figure 8 as distribution of the discrepancies calculated with the expression (3). Both the mean values and the standard deviations shows that the lack of GCPs did not significantly affect the final reconstruction of the 3D model of the coastal section. If compared to the results obtained through the dataset of nadiral imageries, these latter histograms and statistics show that the standard deviation was slightly higher with oblique images for all the analysed cases, while the mean values of the discrepancies are comparable using a set of well-distributed GCPs. The bias around $2 \mathrm{~cm}$ in the mean value was probably due to the delay between the first VPs acquired and the last ones, as previously explained, while an actual difference (again around $2 \mathrm{~cm}$ ) exists for the cases of a single GCP with nadiral imageries.

\section{CONCLUSIONS}

The recent availability of commercial drones with an on-board RTK enables the direct georeferencing of images with 


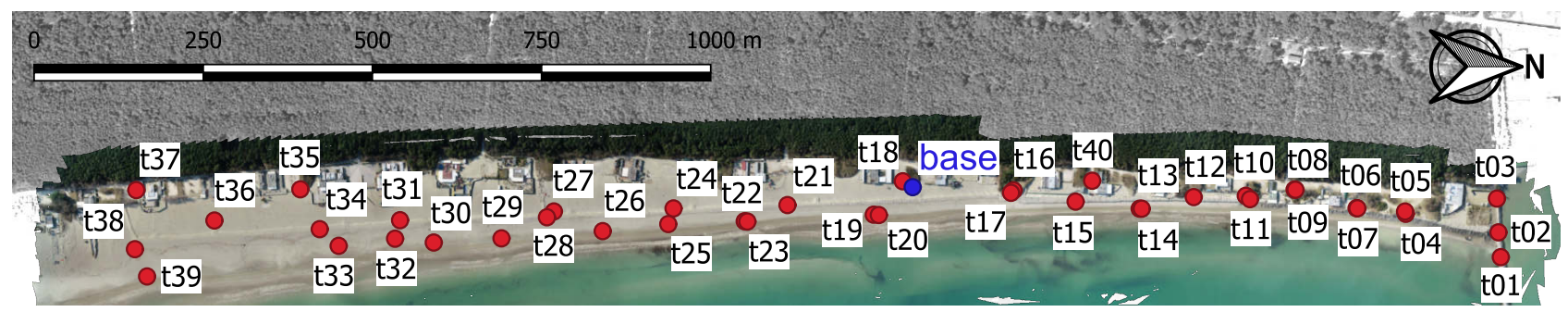

Figure 6. Ground Control Points (red) and base receiver (blue) locations.

Table 3. Summary of the residuals for nadiral imageries with RTK camera coordinates and global ENU standard deviations $(5 \mathrm{~cm})$. Abbreviations are explained in (1) and (2).

\begin{tabular}{|c|c|c|c|c|c|c|c|}
\hline \multirow{2}{*}{\multicolumn{2}{|c|}{ Residuals }} & \multicolumn{5}{|c|}{ Processing Method } & \multirow[b]{2}{*}{ Y-E } \\
\hline & & $\mathrm{N}-\mathrm{S}$ & N-E & 1B-S & $1 \mathrm{P}-\mathrm{S}$ & Y-S & \\
\hline \multirow{3}{*}{ East } & $\operatorname{Min}[\mathrm{m}]$ & -0.009 & -1.004 & $-0.013(-0.001)$ & $-0.014(0.001)$ & $-0.008(-0.012)$ & $-0.091(-0.121)$ \\
\hline & $\operatorname{Max}[\mathrm{m}]$ & 0.035 & 0.448 & $0.030(-0.001)$ & $0.030(0.001)$ & $0.034(0.013)$ & $0.092(0.129)$ \\
\hline & $R M S E[\mathrm{~m}]$ & 0.015 & 0.260 & $0.013(0.001)$ & $0.012(0.001)$ & $0.013(0.006)$ & $0.054(0.060)$ \\
\hline \multirow{3}{*}{ North } & $\operatorname{Min}[\mathrm{m}]$ & -0.057 & -2.678 & $-0.052(-0.005)$ & $-0.052(-0.004)$ & $-0.034(-0.017)$ & $-0.079(-0.082)$ \\
\hline & $\operatorname{Max}[\mathrm{m}]$ & -0.004 & -0.606 & $-0.001(-0.005)$ & $-0.002(-0.004)$ & $0.007(0.006)$ & $0.050(0.057)$ \\
\hline & $R M S E[\mathrm{~m}]$ & 0.027 & 2.025 & $0.022(0.005)$ & $0.021(0.004)$ & $0.016(0.008)$ & $0.042(0.031)$ \\
\hline \multirow{3}{*}{ Up } & $\operatorname{Min}[\mathrm{m}]$ & -1.837 & -0.682 & $-0.014(-0.002)$ & $-0.058(-0.003)$ & $-0.044(-0.022)$ & $-0.390(-0.352)$ \\
\hline & $\operatorname{Max}[\mathrm{m}]$ & -1.719 & 0.847 & $0.068(-0.002)$ & $0.020(-0.003)$ & $0.038(0.028)$ & $-0.061(-0.090)$ \\
\hline & $R M S E[\mathrm{~m}]$ & 1.785 & 0.256 & $0.030(0.002)$ & $0.028(0.003)$ & $0.020(0.012)$ & $0.288(0.223)$ \\
\hline \multirow{3}{*}{$3 D$} & $\operatorname{Min}[\mathrm{m}]$ & 1.719 & 0.895 & $0.005(0.005)$ & $0.005(0.005)$ & $0.002(0.005)$ & $0.071(0.098)$ \\
\hline & $\operatorname{Max}[\mathrm{m}]$ & 1.838 & 2.681 & $0.073(0.005)$ & $0.077(0.005)$ & $0.056(0.029)$ & $0.393(0.359)$ \\
\hline & $R M S E[\mathrm{~m}]$ & 1.785 & 2.057 & $0.040(0.005)$ & $0.037(0.005)$ & $0.029(0.015)$ & $0.296(0.233)$ \\
\hline
\end{tabular}

Table 4. Summary of the residuals for oblique imageries with RTK camera coordinates and Exif recorded ENU standard deviations. Abbreviations are explained in (1) and (2)

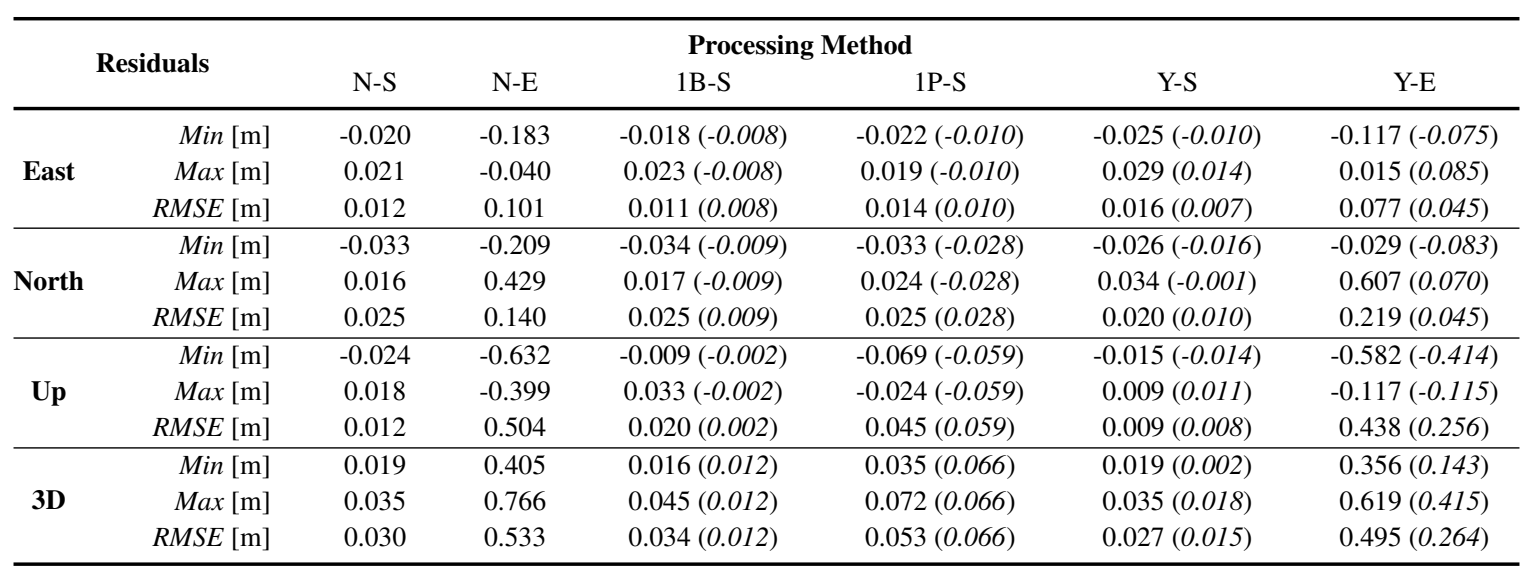

centimetre-level accuracies. The overall cost of these mapping system is rapidly decreasing and many field of application may take advantage in using these newer solutions.

In this work we tested the DJI Phantom 4 RTK combined with its own base receiver, the D-RTK 2 Mobile Station. The system is practically ready-to-use by UAV operators and allows them to acquire dual-frequency RTK accurate images. In addition, the coordinates stored within the metadata are already corrected for both the offset between the APC and camera's perspective centre as well as for the tilt of the drone during the flight.

We highlighted the accuracies of the models by using both nadiral and oblique imageries, following different strategies. The data processing options that were considered included the complete lack of any Ground Control Point, the use of a single GCP to improve the estimation of the focal length and the use of known calibration parameters provided within the Exif metadata.

A first assessment was performed on the basis of control points consisting in signalised markers that were surveyed through a GNSS receiver in NRTK mode, similarly to the collection of GCPs coordinates. A more in-depth analysis was performed through a set of validation points that were not signalised, but simply surveyed on the beach using a plate under the pole to prevent vertical offsets. In this case, a DEM was generated for the cases that proved to be advantageous in term of minor in-situ operations and/or accurate on a CPs basis. 

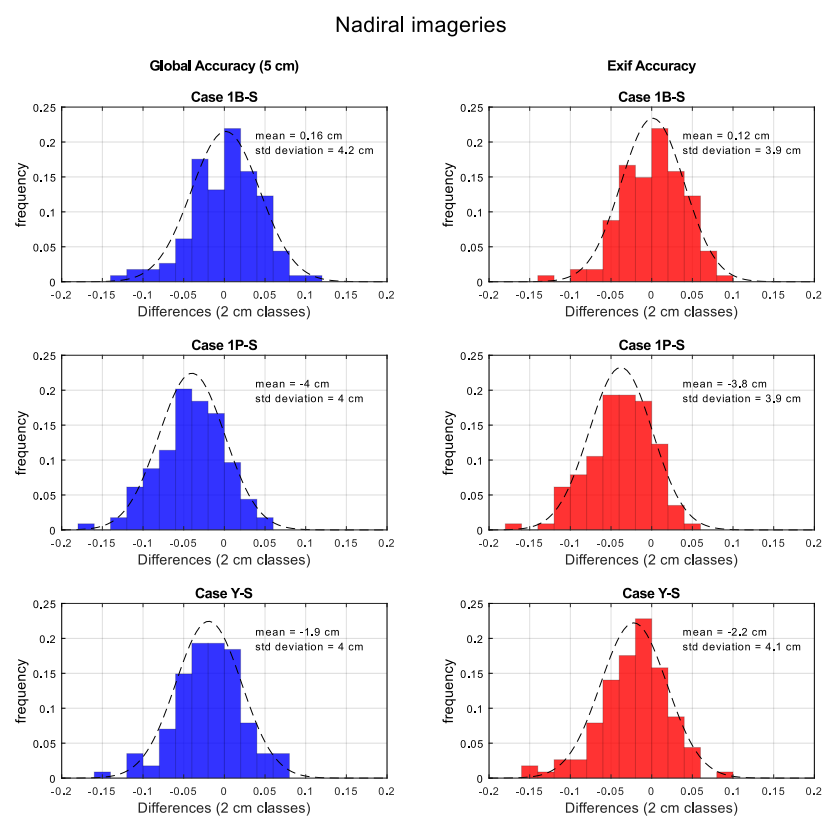

Figure 7. Distribution of the discrepancies computed on the validation points for the nadiral imagery dataset.
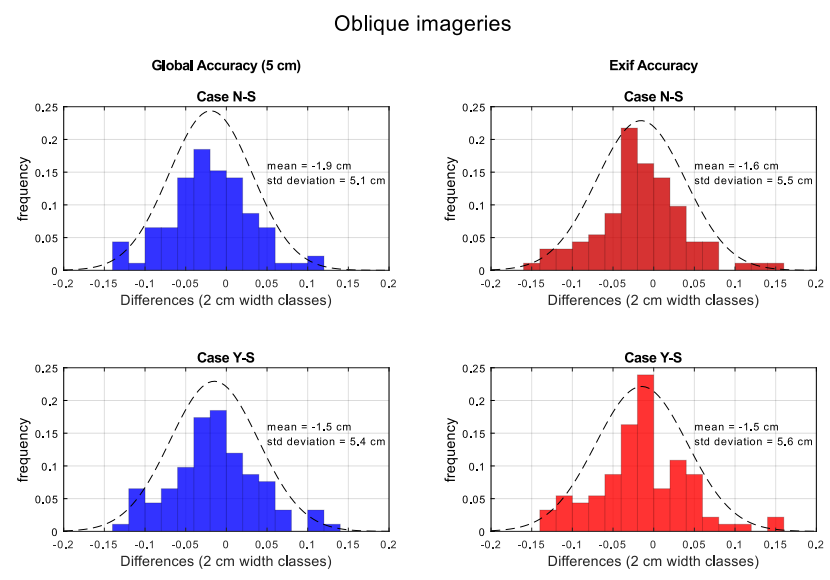

Figure 8. Distribution of the discrepancies computed on the validation points for the oblique imagery dataset.

The analysis of the results showed that the most likely beneficial strategy with the equipment used for the tests in this work is using a single GCP if a nadiral imageries acquisition is planned. In case the area to be mapped would not be accessible for certain reasons, not only for coastal applications but also concerning polluted of hazardous sites, the GCP can be surveyed on the edge of the flight plan with still good final accuracies of the model. Alternatively, the acquisition of oblique imageries proved to provide a reliable estimation of all of the interior orientation's parameters and very good final accuracies even with no GCP. Of course, the number of images to be both acquired and further processed is higher (practically double) and may influence the overall performance in terms of time needed to generate the final model.

However, in this work the base station was approximately placed in the centre of the region to be acquired: even if no GCP has to be surveyed, the base station must be placed in any case. This latter consideration applies especially to inaccessible areas. To overcome this issue it is possible to use an alternative approach to estimate the location of the drone at the time of each image capture based on a Post Processing Kinematic (PPK). For this reason, we are also analysing the benefits of such method for the same datasets presented in this work.

\section{REFERENCES}

Cramer, Michael, Stallmann, D, 2001. System Calibration for Direct Georeferencing. Int. Arch. Photogramm. Remote Sens. Spat. Inf. Sci., 34.

Forlani, Gianfranco, Dall'Asta, Elisa, Diotri, Fabrizio, Cella, Umberto Morra di, Roncella, Riccardo, Santise, Marina, 2018. Quality Assessment of DSMs Produced from UAV Flights Georeferenced with On-Board RTK Positioning. Remote Sensing, 10.

Gabrlik, Petr, 2015. The Use of Direct Georeferencing in Aerial Photogrammetry with Micro UAV. IFAC-PapersOnLine, 48, 380 - 385. 13th IFAC and IEEE Conference on Programmable Devices and Embedded Systems.

Gabrlik, Petr, Jelinek, Ales, Janata, Premysl, 2016. Precise Multi-Sensor Georeferencing System for Micro UAVs. IFAC-PapersOnLine, 49, 170 - 175. 14th IFAC Conference on Programmable Devices and Embedded Systems PDES 2016.

Heipke, C.; Jacobsen, K.; Wegmann H.; Andersen Ø.; Nilsen B., 2000. INTEGRATED SENSOR ORIENTATION - AN OEEPE TEST. International Archives of the Photogrammetry, Remote Sensing and Spatial Information Sciences, XXXIII, 373 - 380.

Martínez-Carricondo, Patricio, Agüera-Vega, Francisco, Carvajal-Ramírez, Fernando, Mesas-Carrascosa, Francisco-Javier, García-Ferrer, Alfonso, Pérez-Porras, Fernando-Juan, 2018. Assessment of UAV-photogrammetric mapping accuracy based on variation of ground control points. International Journal of Applied Earth Observation and Geoinformation, 72, 1 - 10.

Padró, Joan-Cristian, Muñoz, Francisco-Javier, Planas, Jordi, Pons, Xavier, 2019. Comparison of four UAV georeferencing methods for environmental monitoring purposes focusing on the combined use with airborne and satellite remote sensing platforms. International Journal of Applied Earth Observation and Geoinformation, 75, 130 - 140.

Rabah, M., Basiouny, M., Ghanem, E., Elhadary, A., 2018. Using RTK and VRS in direct geo-referencing of the UAV imagery. NRIAG Journal of Astronomy and Geophysics, 7, $220-226$.

Shen, Xiang, Zhang, Yongjun, Li, Qingquan, 2015. Accurate direct georeferencing of aerial imagery in national coordinates. ISPRS Journal of Photogrammetry and Remote Sensing, 105, $13-18$. 